\title{
Shock Wave in Condensed Matter Generated by Impulsive Load
}

\author{
S. I. Anisimov and V. A. Kravchenko \\ L. D. Landau Institute for Theoretical Physics, Academy of Sciences, Moscow, USSR
}

Z. Naturforsch. 40 a, 8-13 (1985); received September 29, 1984

\begin{abstract}
A shock wave in condensed matter generated by impulsive load ("shock loading") is considered. A self-similar solution of the problem is presented. The media are described by the equation-of-state of the Mie-Grüneisen type. Values of the self-similarity exponent and the profiles of gas-dynamical variables have been calculated. The problem of generation of shock waves by ultra-short laser pulses is discussed.
\end{abstract}

\section{Introduction}

The basic experimental information on the thermodynamic properties of condensed matter in the megabar pressure range has been obtained by dynamic methods. These methods are based on measuring the parameters of matter behind a plane stationary shock wave and on calculating the pressure and internal energy using the laws of conservation of mass, momentum and energy [1]. Along with traditional methods of strong shock wave generation by means of explosives and light-gas guns the above methods allow investigating in detail the thermodynamic properties of a great number of substances under pressures of up to $\sim 5 \mathrm{Mbar}$. Application of powerful lasers and relativistic electron beams makes it possible to obtain much higher pressures. However, in this case the experiments with plane stationary shock waves become rather complicated, since the required laser energy increases rapidly with pressure [2]. At the same time very complicated gas-dynamic numerical simulations are needed to get thermodynamic information from experiments with variable intensity shock waves. The calculations are simpler for the case when the hydrodynamic motion of matter is self-similar. Of the corresponding solutions of hydrodynamic equations the second kind self-similar ones $[3,4]$ are of particular interest, since for them the self-similarity exponent depends on the thermodynamic properties of a medium, and even simple measurements of the shock front law of motion may produce information on the equation of state.

Note that an example of the second kind selfsimilar solution for a thermodynamically non-ideal medium is given in [5], where the problem of spherical shock collapse is considered. The selfsimilarity exponent is found to depend on the equation of state of a medium.

In what follows we consider an example of selfsimilar flow of condensed matter, which is interesting from the experimental point of view: a motion induced by impulsive load ("shock loading"). Such a flow arises particularly when a short laser pulse acts upon the medium [6]. The problem of "shock loading" has been studied in detail for the case when the medium is a cold ideal gas (see [3] and references therein). This problem will be solved for a medium described by the Mie-Grüneisen equation-of-state (EOS), and the dependence of the selfsimilarity exponent on the EOS parameters will be found. The technique employed allows consideration of a more general EOS and may turn out useful for analyzing the interaction of ultra-short laser pulses with matter.

\section{Formulation of the Problem}

Suppose a medium having the equation-of-state

$$
p=\varrho \varepsilon \Gamma\left(\varrho / \varrho_{0}\right),
$$

where $\varepsilon$ is the specific internal energy, $\Gamma$ the Grüneisen coefficient and $\varrho_{0}$ the initial density, occupies the half-space $x>0$. For some time $\tau_{0}$ the boundary $x=0$ of the half-space is subjected to the impulsive loading with the pressure amplitude $p_{0}$. The motion of the medium at times $t \gg \tau_{0}$ is to be determined. Such a formulation corresponds exactly to that described in [3]. The only difference is in the equation of state. The question of a relationship of the quantities $p_{0}$ and $\tau_{0}$ with laser pulse parameters,

0340-4811 / $85 / 0100-0008 \$ 01.30 / 0$. - Please order a reprint rather than making your own copy. 
which is most essential for the laser method of excitation of shock waves, will be considered below in Section 5.

A shock wave arises in matter as a result of impulsive loading. Suppose $X=X(t)$ and $D=\dot{X}(t)$ are the coordinate and velocity of the shock wave front. Putting in the equations of hydrodynamics

$$
\begin{aligned}
& \varrho(x, t)=\varrho_{0} G(\xi), \quad u(x, t)=D v(\xi), \\
& p(x, t)=\varrho_{0} D^{2} \Pi(\xi), \quad \xi=x / X(t)
\end{aligned}
$$

and using the equation of state (1) it is easy to see that the variables are separated, provided $X(t)$ $=A t^{\alpha}$. The self-similarity exponent $\alpha$ should be determined in the process of solving the problem. The constant $A$ is related to the parameters of a "shock loading" $p_{0}$ and $\tau_{0}$, and its explicit form is involved in the law of the shock wave motion only. Further it is convenient to introduce new variables:

$$
V(\xi)=\xi-v(\xi), \quad Z(\xi)=\Pi(\xi) / G(\xi) .
$$

After subsitution of these variables into the equations of gas-dynamics we obtain a set of ordinary differential equations

$$
\begin{aligned}
& V^{\prime}+V(\ln G)^{\prime}=1, \\
& V^{\prime} V+Z(\ln G)^{\prime}+Z^{\prime}=V(1-\mu)+\mu \xi, \\
& \varphi(G)(\ln G)^{\prime}+(\ln Z)^{\prime}=-2 \mu / V,
\end{aligned}
$$

where

$$
\varphi(G)=-\Gamma(G)-\frac{\partial \ln \Gamma(G)}{\partial \ln G}, \quad \mu=\frac{1}{\alpha}-1
$$

and the primes indicate derivatives with respect to $\xi$. The boundary conditions for a strong shock wave, $\xi=1$, can be given as follows:

$$
V(1)=\frac{1}{\beta}, \quad G(1)=\beta, \quad Z(1)=\frac{\beta-1}{\beta^{2}} .
$$

The value of the limiting compression $\beta$ is found from the solution of the equation

$$
\Gamma(\beta)(\beta-1)=2,
$$

which follows directly from the Rankine-Hugoniot relation.

The solution of (2) should also satisfy the obvious conditions

$$
\Pi(\xi) \rightarrow 0, \quad G(\xi) \rightarrow 0 \text { for } \xi \rightarrow-\infty .
$$

\section{Solution of Equations (2)}

The system of equations (2) with boundary conditions (3), (5) should be integrated numerically. The parameter $\mu$ is the eigenvalue of the system and is determined in the process of its integration from the conditions of uniqueness of the solution. The latter can be formulated as a requirement that the integral curve should pass through a certain singular point of system (2). In [3] an estimation of variation range of the exponent $\alpha$ is presented: $1 / 2<\alpha<2 / 3$. It is independent of thermodynamic properties of the medium and holds for the case under consideration. For the eigenvalue $\mu$ the following inequality follows: $1 / 2<\mu<1$.

Combining the first and third equations of system (2), it is easy to obtain an integral, which is usually called the adiabaticity integral [7]. For the EOS (1) it has the form

$$
Z(G V)^{2 \mu} f(G)=J=\text { const },
$$

where

$$
F(G)=\frac{1}{\Gamma(G)} \exp \left[-\int \Gamma(G) \mathrm{d} \ln G\right] .
$$

The value of the constant $J$ is determined from the boundary conditions (3):

$$
J=\frac{f(\beta)(\beta-1)}{\beta^{2}} .
$$

The problem is thus reduced to integration of a system of two ordinary differential equations. It should be noted that in the earlier mentioned case of an ideal gas $(\varphi=$ const $)$ the problem of obtaining a self-similar solution allows further simplification and has been finally reduced to a single ordinary differential equation and quadratures. In our case such a simplification is impossible and the technique of solution should be different.

Integration of system (2) can be easily performed in the following way. Let us resolve system (2) with respect to the derivatives

$$
\frac{\mathrm{d} \ln G}{\mathrm{~d} \xi}=\frac{\Delta_{1}}{\Delta}, \frac{\mathrm{d} V}{\mathrm{~d} \xi}=\frac{\Delta_{2}}{\Delta}, \quad \frac{\mathrm{d} Z}{\mathrm{~d} \xi}=\frac{\Delta_{3}}{\Delta},
$$

where

$$
\begin{aligned}
& \Delta=V\left\{Z[1-\varphi(G)]-V^{2}\right\}, \Delta_{1}=\mu[2 Z-V(V-\xi)], \\
& \Delta_{2}=\Delta+V \Delta_{1}, \quad \Delta_{3}=\mu(\xi-V) \Delta+\left(V^{2}-Z\right) \Delta_{1} .
\end{aligned}
$$


The determinant $\Delta$ in the space of variables $(G, V, Z)$ becomes zero on the surface $Z=V^{2}$. $[1-\varphi(G)]^{-1}$. The intersections of this surface by the planes $G=$ const are parabolas passing through the origin (Figure 1). For the solution to be unique the integral curve should cross the surface $\Delta=0$ only at the points where the determinants $\Delta_{1}, \Delta_{2}, \Delta_{3}$ are zero too, i.e., at singular points of system (6).

We show that the integral curve satisfying the boundary conditions (3) and (5) should necessarily cross the surface $\Delta=0$. For this purpose we consider the relative positions of the point, determined by the boundary conditions (3), and of the nonuniqueness parabola $Z=V^{2}[1-\varphi(G)]^{-1}$, which is located in the plane $G=\beta$. It can be seen that the point corresponding to the shock wave front is to the left of "its own" non-uniqueness parabola, provided the condition

$$
\beta[1-\varphi(\beta)]>2-\varphi(\beta)
$$

is satisfied. Using the determination of the function $\varphi(G)$ and the Rankine-Hugoniot equation this inequality can be transformed to

$$
\frac{\mathrm{d}}{\mathrm{d} G}\left[\frac{2}{\Gamma(G)}\right]_{G=\beta}<1 .
$$

Condition (8) is not a thermodynamical inequality but many functions $\Gamma(\varrho)$, which are of practical interest, satisfy it. In what follows we assume that condition (8) is fulfilled*.

Now we consider the asymptotic behaviour of the solution at $\xi \rightarrow-\infty$. Assuming $V(\xi) \sim c_{1} \xi$, $Z(\xi) \sim c_{2}(-\xi)^{k}, \quad G(\xi) \sim c_{3}(-\xi)^{-1}, \quad$ substituting this into (2) and (6) and taking into account of the boundary condition (5) we get

$$
\begin{aligned}
& c_{1}=-\mu, \quad k=2+v l, \quad l=1+\mu^{-1}, \\
& c_{2} c_{3}^{2 \mu+v} \mu^{2 \mu} v^{-1}=-J, \quad v=\varphi(0)<0 .
\end{aligned}
$$

It is clear that at $\xi \rightarrow-\infty$ the integral curve is tangential to the plane $G=0$ and has a smaller inclination angle to the axis $V$ than any of the parabolas $Z=V^{2}[1-\varphi(G)]^{-1}$. Hence, it follows that some part of the integral curve in the plane $G=0$ is more shifted to the right (that is, it is located at larger values of $V$ ) than any of non-uniqueness parabolas

\footnotetext{
* Formally, inequality (8) is connected with the condition of uniqueness of solution of (4) for the compression ratio in a shock wave.
}

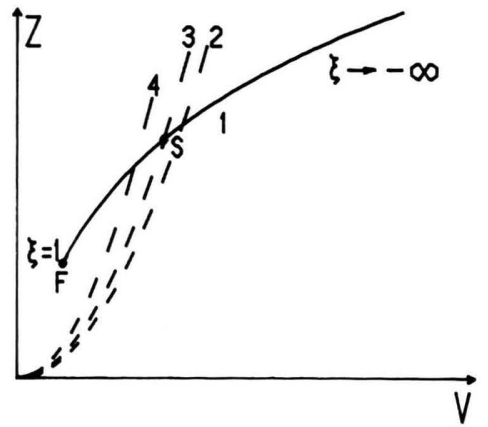

Fig. 1. Projection of the integral curve on the $(Z, V)$-plane, 1 integral curve; $2-4$ parabolas of non-uniqueness in planes $G=$ const; F projection of the shock wave front; $\mathrm{S}$ projection of the singular point.

(Figure 1). Thus, on an integral curve there are points lying on different sides of the surface $\Delta=0$.

Let $G^{*}, V^{*}, Z^{*}, \xi^{*}$ be the values of the variables at the intersection point of the integral curve with the surface $\Delta=0$. As we have mentioned above, the point of intersection should be a singular point of system (7), in which all the determinants $\Delta_{k}$ become zero. Together with condition $\Delta=0$ this gives two relations between the variables at the singular point:

$$
\begin{aligned}
& Z=V^{2}[1-\varphi(G)]^{-1}, \\
& Z=\frac{1}{2} V(V-\xi),
\end{aligned}
$$

since the determinants $\Delta_{2}$ and $\Delta_{3}$ are linear combinations of $\Delta$ and $\Delta_{1}$. The third independent equation is given by the adiabaticity integral (6). So, conditions (6) and (10) are not sufficient for determining the values of all the variables at a singular point, and, unlike the ordinary case of an ideal gas $[3,4,8]$, the problem of finding an integral curve is double-parametric: the values of the variables $G^{*}, V^{*}, Z^{*}$ and $\xi^{*}$ at a singular point should, as well as the parameter $\mu$, be determined in the process of solving the problem. The algorithm of the solution is as follows. Putting the trial values of $\mu$ and one of the variables at the singular point $\left(G^{*}\right.$ is most convenient) we calculate the values of $V^{*}$, $Z^{*}, \xi^{*}$ using (6) and (10). Integrating then (7) from the singular point to the shock wave front, we choose a pair of values of $\mu$ and $G^{*}$ for which the solution at $\xi=1$ satisfies conditions (3) on the shock wave.

To start the numerical calculation from the singular point, one should construct the expansion of all the variables into series in the vicinity of $\xi=\xi^{*}$. 
Equations (7) in the explicit form can be written out as follows:

$$
\begin{aligned}
& \mathrm{d}(\ln G) / \mathrm{d} \xi=F, \\
& \mathrm{~d} V / \mathrm{d} \xi=1-V F, \\
& \mathrm{~d} Z / \mathrm{d} \xi=\mu(\xi-V)+\left(V^{2}-Z\right) F,
\end{aligned}
$$

where

$$
F=\frac{\mu}{V} \frac{2 Z-V(V-\xi)}{[1-\varphi(G)] Z-V^{2}} \equiv \frac{\Delta_{1}}{\Delta} .
$$

In the vicinity of the singular point we put

$$
\begin{aligned}
& V(\xi) \cong V^{*}+k_{1} \eta, \quad Z(\xi) \cong Z^{*}+k_{2} \eta, \\
& \ln G(\xi) \cong \ln G^{*}+k_{3} \eta, \quad \eta=\xi-\xi^{*} .
\end{aligned}
$$

Substituting it into (11) we get the quadratic equation for $k_{3}$

$$
M k_{3}^{2}+N k_{3}+Q=0,
$$

where

$$
\begin{aligned}
& M=2-\varphi\left(G^{*}\right)-G^{*}\left(\frac{\mathrm{d} \varphi}{\mathrm{d} G}\right)_{G=G^{*}} \cdot\left[1-\varphi\left(G^{*}\right)\right]^{-1}, \\
& N=-\frac{5 \mu+2}{V^{*}}, \quad Q=\frac{2 \mu(2 \mu+1)}{V^{* 2}\left[1-\varphi\left(G^{*}\right)\right]} .
\end{aligned}
$$

In the cases considered below the solution of the problem corresponds to the greater root of (12).

\section{Discussion of the results}

Consider as an example the equation of state (1) with the Grüneisen coefficient in the following form

$$
\Gamma(G)=\frac{2}{3}+\left(\Gamma_{0}-\frac{2}{3}\right) \cdot \frac{G_{m}^{2}+1}{G_{m}^{2}+G^{2}} \cdot G
$$

where $\Gamma_{0}$ and $G_{\mathrm{m}}$ are the parameters which are usually determined from the experiment. Equation (1) with the function $\Gamma(G)$ determined by (13) provides a proper qualitative description of the thermal component of the pressure in metals in a wide range of pressures and has correct asymptotics at $G \rightarrow 0$ and $G \rightarrow \infty$. The parameter $\Gamma_{0}$ is the Grüneisen coefficient under normal conditions. It equals 1.78 for iron, 2.02 for copper and 2.19 for aluminium. The parameter $G_{m}$ lies in the range between 0.5 and 0.8 . The experiments with shock waves generated by "impulsive load" make it possible in principle to elucidate the value of $G_{m}$ by comparing the experimental value of $\alpha$ with those calculated for different values of $G_{m}$.

The results of solving the problem using the approach described in the previous Section are presented in a table and in Figures $2-4$. The value of the exponent $\alpha$ for an ideal gas with $\Gamma=2 / 3$, obtained by the above method, is also given in the table. The variation of the values of $\alpha$ obtained for different values of $\Gamma_{0}$ and $G_{m}$ is rather noticeable if account is taken that all the range of the parameter $\alpha$ variation is 0.17 .

The calculations show that variation of the EOS affects essentially the profiles of hydrodynamical variables, the value of the limiting compression and the singular point parameters. Fig. 2 shows the inte-

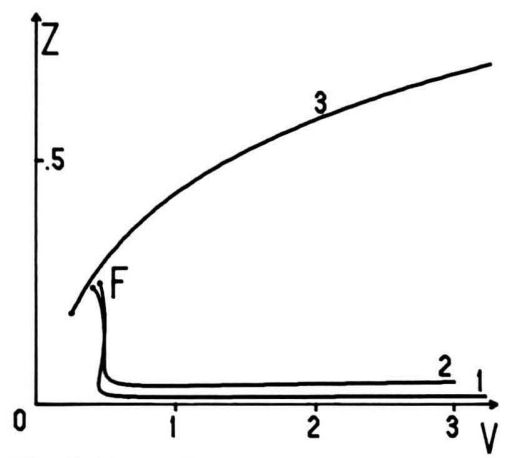

Fig. 2. Integral curves in the $Z V$-plane. 1 EOS (1), (13); $\Gamma_{0}=2.19 ; G_{m}=0.8 ; 2 \operatorname{EOS}(1),(13) ; \Gamma_{0}=1.78 ; G_{m}=0.8 ;$ 3 ideal gas; $\Gamma=2 / 3=$ const.

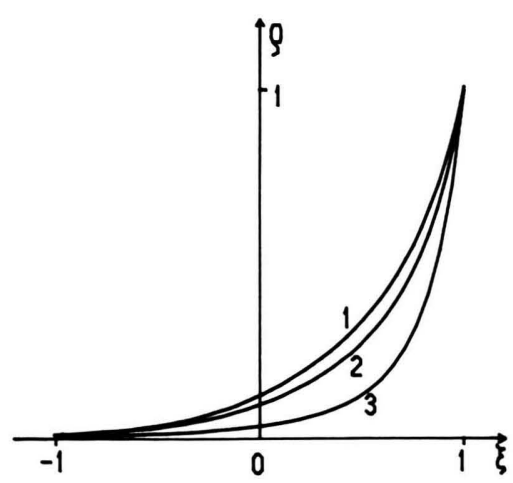

Fig. 3. Normalized density as a function of $\xi$. $1 \Gamma_{0}=2.19$, $G_{m}=0.8 ; 2 \Gamma_{0}=1.78, G_{m}=0.8 ; 3 \Gamma=2 / 3=$ const. 

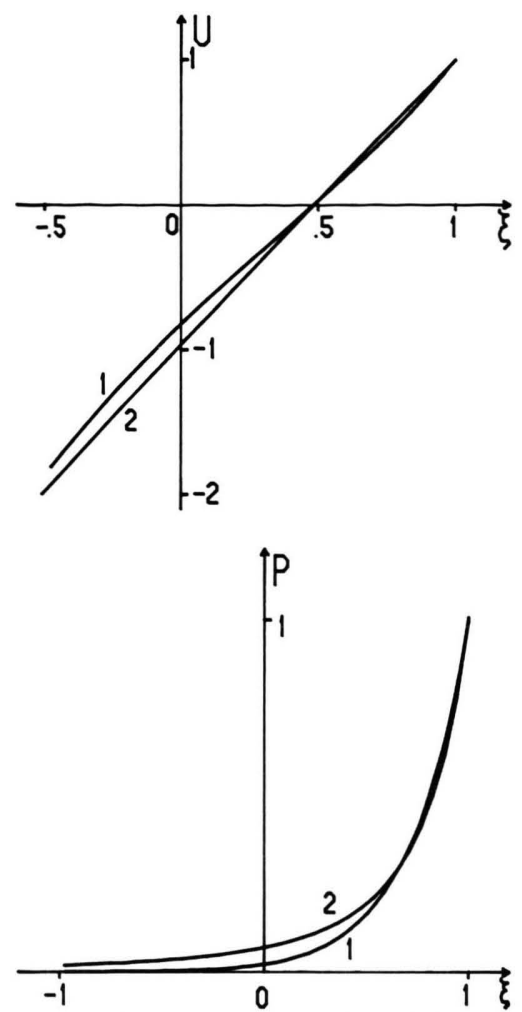

Fig. 4. Normalized velocity (4a) and pressure (4b) as functions of $\xi .1 \Gamma_{0}=2.19, G_{m}=0.8 ; 2 \Gamma=2 / 3=$ const.

Table

\begin{tabular}{lllllr}
\hline$\Gamma_{0}$ & $G_{m}$ & $\alpha$ & $\beta$ & $G^{*}$ & \multicolumn{1}{l}{$\xi^{*}$} \\
\hline \multirow{2}{*}{1.78} & 0.50 & 0.6304 & 2.722 & 0.356 & 0.196 \\
& 0.65 & 0.6288 & 2.617 & 0.346 & 0.170 \\
& 0.80 & 0.6273 & 2.506 & 0.335 & 0.148 \\
2.02 & 0.50 & 0.6321 & 2.525 & 0.392 & 0.214 \\
& 0.65 & 0.6305 & 2.420 & 0.381 & 0.189 \\
2.19 & 0.80 & 0.6289 & 2.312 & 0.371 & 0.168 \\
& 0.50 & 0.6331 & 2.402 & 0.415 & 0.223 \\
& 0.65 & 0.6312 & 2.299 & 0.404 & 0.199 \\
$\Gamma=2 / 3=$ & 0.80 & 0.6311 & 2.197 & 0.401 & 0.180 \\
& & & &
\end{tabular}

gral curves in the plane $(Z, V)$. For an ideal gas at all values of $\xi$ the pressure drops with decreasing $\xi$ more slowly than the density, and the value of $Z \sim p / Q$ increases thus monotonously with $V$. For a medium with equation of state (1) in the vicinity of the shock wave front an opposite situation is observed: the pressure drops faster than the density.
But at $\xi$ of the order of $-3 \div-5$ or less the medium, as is shown by calculation, becomes sufficiently "ideal" and the value of $Z$ begins to grow slowly. Figures 3 and 4 show the dependences of the density, velocity and pressure of some substance on the variable $\xi$. All the quantities given are divided by their values at the shock wave front. The density profiles for the equation of state (1) (curves 1,2) differ noticeably from the profile for an ideal gas (curve 3 ). The profiles of velocity (Fig. $4 \mathrm{a}$ ) and pressure (Fig. 4 b) are less sensitive to variation of EOS.

\section{On excitation of shock waves by ultra-short laser pulses}

Now we consider how the parameters $p_{0}$ and $\tau_{0}$, determining the shock wave amplitude for the "impulsive load", are related to the parameters of the laser pulse. Suppose the absorbed laser energy (per a unit area) is $Q$, and the duration of the laser pulse $\tau$ is much shorter than all the other characteristic times of the problem (the corresponding inequality will be given below)* The absorbed radiation energy is transferred to electrons. The ions for the time of the order of the relaxation time $\tau_{\mathrm{ei}}$ remain cold, and hydrodynamical motion does not contribute to the energy transfer. Energy is transferred inside cold matter by electron heat conductivity. Due to strong temperature dependence of the heat conductivity coefficient this process is a kind of a thermal wave with a sharp front and a practically rectangular spatial temperature profile. A simple estimation (see, for instance, [9]) shows that hydrodynamical perturbations reach the thermal front for a time of the order of

$$
\tau_{\mathrm{H}} \sim A^{3 / 4} Z^{-3 / 2} Q^{1 / 2} \text {. }
$$

Here $A$ is the mass number of the substance and $Z$ the ion charge, the time being measured in picoseconds, $Q$ in $\mathrm{J} / \mathrm{cm}^{2}$; the ion number density is assumed to equal $6 \cdot 10^{22} \mathrm{~cm}^{-3}$. At the times exceeding $\tau_{\mathrm{H}}$ the expansion of the substance into vacuum becomes essential, and the pressure in a perturbed substance decreases rapidly. It is clear that $\tau_{\mathrm{H}}$ in the

* Everywhere below $Q$ means the absorbed laser energy. The problem of the mechanisms of absorption and the fraction of the energy absorbed are beyond the framework of the present paper. 
case of laser shock wave generation plays the role of the loading time $\tau_{0}$. The pressure amplitude (in Mbars) is of the order of magnitude

$$
p_{\mathrm{H}} \sim Z^{4 / 3} A^{-1 / 6} Q^{1 / 3} .
$$

This value plays the role of $p_{0}$. For the condition of "shock loading" to be fulfilled, the duration of the laser pulse should be much shorter than $\tau_{\mathrm{H}}$.

In the experiments under discussion pressures of the order of 10 Mbars can be obtained when the

[1] L. V. Al'tshuler, Uspekhi Fiz. Nauk 85, 197 (1965).

[2] S. I. Anisimov, A. M. Prokhorov, and V. E. Fortov, Uspekhi Fiz. Nauk 142, 395 (1984).

[3] Ya. B. Zel'dovich and Yu. P. Raizer, Physics of Shock Waves and High Temperature Hydrodynamic Phenomena, Academic Press, New York 1967.

[4] G. I. Barenblatt, Similarity, Selfsimilarity, and Intermediate Asymptotics, Consultants Bureau, New York 1979.

[5] R. A. Axford and D. D. Holm, Proc. 2-nd Int. Symp. on Group-Theor. Methods in Mechanics, Novosibirsk, USSR, 1978, p. 48. value of $Q$ is about several $\mathrm{J} / \mathrm{cm}^{2}$. The pulse duration in this case should be less than $\sim 10$ psec. The total laser energy required for such an experiment does not exceed 100 Joules.

\section{Acknowledgements}

The authors are grateful to A. V. Bushman for advice in choosing the approximation for the Grüneisen coefficient and to L. N. Shchur for help with numerical calculation.

[6] S. I. Anisimov, Zh. Experim. i Teor. Fiz. 58, 337 (1970).

[7] L. I. Sedov, Similarity and Dimensional Methods in Mechanics, Academic Press, New York 1959.

[8] J. Meyer-ter-Vehn and C. Schalk, Z. Naturforsch. 37 a, 955 (1982).

[9] S. I. Anisimov, Ya. A. Imas, G. S. Romanov, and Yu. V. Khodyko, Action of High-Power Radiation on Metals, Natl. Techn. Inform. Service, Springfield, Va. 1971 